\title{
EDITORIAL
}

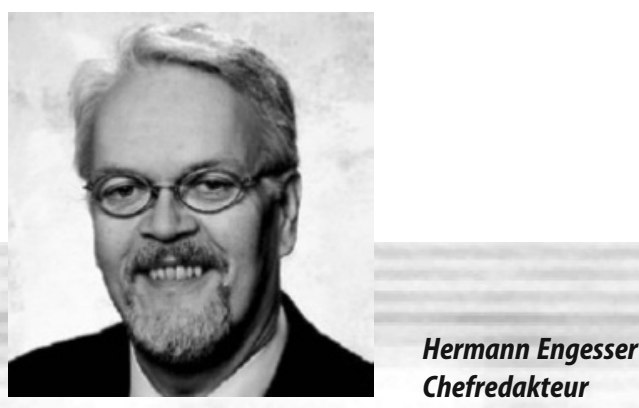

\section{Next Level}

Am Beispiel der Next-Level-Strategie von ABB beschreibt Christopher Ganz Entwicklungspfade hin zum Internet der Dinge, Dienstleistungen und Menschen in einem global agierenden Unternehmen. Sein Fazit lautet: Die nächste Stufe der industriellen Entwicklung erfordert eine ganzheitliche Sichtweise, eine umfassende Aus- und Fortbildung der Mitarbeiter und die Anwendung moderner Technologien mit Digitalisierung und Internet-Anbindung auf alle Bereiche der Industrie.

Bei disruptiven Geschäftsmodellen können erfolgreiche Produkte oder Dienstleistungen durch Digitalisierung völlig neu definiert werden. Vorhandene Anbieter können dabei ihre wirtschaftlichen und technischen Kompetenzen verlieren. Neue Anbieter können in den Markt eintreten und die bisher erfolgreichen verdrängen. August-Wilhelm Scheer erläutert in seinem Beitrag Nutzentreiber der Digitalisierung - ein systematischer Ansatz zur Entwicklung disruptiver digitaler Geschäftsmodelle anhand verschiedener Beispiele dass disruptive Modelle dazu führen können, dass bestehende, traditionsreiche Großunternehmen nicht sicher sein können, dass sie im Rahmen der Digitalisierung ihre Vormachtstellung behalten werden und aufkommende digitale Produkte lediglich als Ergänzung ihrer Leistungen ansehen können. Garagengründungen können es in kurzer Zeit durch extremes Wachstum schaffen, bestehende Weltmarktführer in ihren Marktpositionen zu erschüttern oder sogar zu verdrängen.

Daniel Sonntag et al. stellen in ihrem Beitrag The Clinical Data Intelligence Project ein Projekt vor, bei dem durch Kombination und Verknüpfung von Daten und Methoden die medizinische Betreuung individualisiert werden kann.

Die Begriffe Digitalisierung und Industrie 4.0 werden gegenwärtig mit unterschiedlichen Inhalten besetzt. Das mag ein Indiz sein, dass ein v. a. im deutschsprachigen Raum sehr begrüßenswerter Trend durch Modewellen überlagert wird. Es könnte das Verlaufsmuster des Gartner Hype Cycle beobachtet werden. Dieser Verlauf impliziert Ressourcenvergeudung. Peter Mertens und Dina Barbian stellen in Ihrem Artikel Digitalisierung und Industrie 4.0 - Trend mit modischer Überhöhung? retardierende Momente zur Diskussion, wie etwa unterschiedliche Kulturen zwischen der Entwicklung von Software und Maschinen oder Probleme mit dem Lebenszyklus von individualisierten Erzeugnissen, die dazu führen könnten, dass man nach dem Gipfel in ein „Tal der Enttäuschungen" stürzt.

Im Aktuellen Schlagwort stellen Christoph Meinel und Julia von Thienen Design Thinking vor, ein Ansatz, der geprägt ist durch die kreative Zusammenarbeit von multidisziplinären Teams in offenen Arbeitsumgebungen, die Methoden verschiedener Fachrichtungen nutzen, um grundlegend sinnvolle Innovationen zu entwickeln.

Der Arbeitsmarkt für IT-Fachleute zeigte sich auch 2015 in einer sehr guten Verfassung, berichten Ralf Beckmann und Susanne Lindner im Forum. Nach wie vor gibt es nur wenig Arbeitslose mit IT-Berufen. Das Vordringen der Informatik in nahezu alle Arbeits- und Lebensbereiche geht einher mit einem überdurchschnittlichen Zuwachs an Arbeitsplätzen für Computerfachleute in den letzten Jahren. Die Nachfrage nach neuen Mitarbeitern bewegte sich, gemessen am Bestand der 2015 bei der Bundesagentur für Arbeit gemeldeten Stellen, auf Höchstniveau. Gleichzeitig hatten Unternehmen Schwierigkeiten, ihre vakanten Stellen für hochqualifizierte IT-Experten zu besetzen. Hier könnte in den kommenden Jahren eine steigende Zahl von Absolventen eines Informatikstudiums zur Entspannung beitragen ebenso wie die zunehmende Attraktivität Deutschlands bei qualifizierten Fachleuten aus dem Ausland.

Viel Lesevergnügen bei den genannten Artikeln und den weiteren Beiträgen.

\section{Hermann Engesser}

DOI $10.1007 /$ s00287-016-0979-0 